\title{
Contemporary imaging methods for the follow-up after endovascular abdominal aneurysm repair: a review
}

\author{
Wojciech Kazimierczak ${ }^{1}$, Zbigniew Serafin ${ }^{1}$, Natalia Kazimierczak ${ }^{2}$, Przemysław Ratajczak ${ }^{1}$, Waldemar Leszczyński ${ }^{1}$, \\ tukasz Bryl ${ }^{1}$, Adam Lemanowicz ${ }^{1}$ \\ ${ }^{1}$ Department of Radiology and Diagnostic Imaging, Nicolaus Copernicus University, Collegium Medicum, Bydgoszcz, Poland \\ ${ }^{2}$ Kujawy and Pomorze University, Bydgoszcz, Poland
}

Videosurgery Miniinv 2019; 14 (1): 1-11 DOI: https://doi.org/10.5114/wiitm.2018.78973

\begin{abstract}
Abdominal aortic aneurysm (AAA) is defined as a localized enlargement of the aortic cross-section where the diameter is greater than $3 \mathrm{~cm}$ or more than $50 \%$ larger than the diameter in a normal segment. The most important complication of AAA is rupture, which, if untreated, results in mortality rates of up to $90 \%$. Conventional open surgical repair is associated with significant 30-day mortality. Endovascular aneurysm repair (EVAR) is a significantly less invasive procedure; it is related to a lower early mortality rate and a lower number of perioperative complications. Although EVAR is a minimally invasive technique, lifelong follow-up imaging is necessary due to possible late complications including endoleak, recurrent aneurysm formation, graft infection, migration, kinking and thrombosis. The total rate of complications after EVAR is estimated at approximately $30 \%$, and the rate of complications that require intervention is 2-3\%. Early detection and progression analysis of such situations is crucial for proper intervention.
\end{abstract}

Key words: endovascular aneurysm repair, abdominal aortic aneurysm, aortic stent graft, endovascular abdominal aneurysm repair.

\section{Introduction}

Abdominal aortic aneurysm (AAA) is defined as a localized enlargement of the aortic cross-section where the diameter is greater than $3 \mathrm{~cm}$ or more than $50 \%$ larger than the diameter in a normal segment. The etiology of AAA is usually complex, involving both environmental and genetic factors. Main AAA risk factors include age over 65, atherosclerosis regardless of localization, male sex, Caucasian ethnicity, smoking, family history, and hypertension $[1,2]$. Most AAAs are located in the infrarenal region while juxtarenal aortic aneurysms account for $15 \%$ of cases [3]. It is estimated that aneurysms occur in $4-8 \%$ of men and $1-2 \%$ of women aged over 65 [4-6]. However, as in most patients the aneurysm remains asymptomatic and its detection is usually incidental, the actual prevalence of AAA is uncertain.

The most important complication of AAA is rupture, which, if untreated, results in mortality rates of up to $90 \%$ [2]. The major risk factors for aneurysm rupture include the diameter at the time of diagnosis, aneurysm size progression, and male gender [7]. Conventional open surgical repair was the gold standard for treatment of AAAs for more than 50 years but is associated with a significant 30-day mortality [8]. Endovascular aneurysm repair (EVAR) was proposed by Volodos et al. in 1987 [9] and then promoted by Parodi et al. [10]. As EVAR is a significantly less invasive procedure than open aneurysm repair, it is related to a lower early mortality rate and low-

\section{Address for correspondence}

Zbigniew Serafin MD, PhD, Department of Radiology and Diagnostic Imaging, Nicolaus Copernicus University, Collegium Medicum,

9 M. Skłodowskiej-Curie St, 85-094 Bydgoszcz, Poland, phone: +48 571377 599, e-mail: serafin@cm.umk.pl 
er number of perioperative complications. Although EVAR is a minimally invasive technique, lifelong follow-up imaging is necessary due to possible late complications. Those include endoleak, recurrent aneurysm formation, graft infection, migration, kinking and thrombosis. The total rate of complications after EVAR is estimated at approximately $30 \%$, and the rate of complications that require intervention is 2-3\% [11]. Early detection and progression analysis of such situations is crucial for proper intervention.

\section{Aim}

The aim of this review was to discuss current imaging methods used for follow-up of patients after EVAR with special attention paid to the invasiveness of procedures.

\section{Common complications of endovascular aneurysm repair}

\section{Endoleak}

Endoleak is a basic and unique complication after EVAR and is a main contributor to aneurysm enlargement and potential rupture. In the classic definition of White et al. endoleak describes incomplete exclusion of the aneurysm sac by the graft [12]. Endoleak is frequent after EVAR and occurs in nearly one in four patients at some time during follow-up [13]. In most cases endoleaks remain asymptomatic. Therefore, lifelong surveillance following EVAR is suggested [14]. Classification of endoleaks is based on the source of blood flow into the space between the graft and the aneurysm wall. Correct classification is important because of different treatment strategies. Five different types of endoleaks are distinguished:

- Type I is related to a leak at the incomplete attachment of the graft to the aortic lumen, which leads to pressure increase in the aneurysm sac; type la proximal, type Ib distal and type Ic related to inadequate seal of iliac occluder. This type of endoleak is associated with high risk of rupture and requires immediate treatment.

- Type II endoleaks are considered most frequent, occurring in approximately $40 \%$ of all leaks [14]. They are caused by branch arteries of the aorta or iliac artery filling the aneurysm sac. Two subtypes are distinguished: Ila with one artery and Ilb with multiple collateral arteries supplying the sac. Treatment is recommended in the case of enlargement of the aneurysm sac by more than $10 \mathrm{~mm}$ [13].

- Type III endoleaks are characterized by EVAR graft mechanical failure. Two subtypes are distinguished: graft rupture (IIla) and fabric disruption (IIIb). Type III endoleaks require active management.

- Type IV endoleaks are usually detected in the early postoperative phase (first 30 days). They are caused by porous flow through the fabric of the graft. Type IV endoleaks are almost never seen in newer generation stent grafts.

- Type $V$ endoleaks refers to endotension, i.e. increase in size of the aneurysm sac without detectable endoleak. Type $V$ endoleaks are idiopathic in nature and diagnosis is based on exclusion of type I-IV endoleaks.

\section{Other complications}

Component separation and further dislocation were more prevalent in the first generation of stent grafts and may be caused by shrinkage of the aneurysm sac and increased pressure towards the graft's iliac limbs. Component separation may result in type III endoleak and device migration [13]. Device migration is a relatively common complication occurring in approximately $3 \%$ of patients [15]. Significant migration is defined as a dislocation of $5-10 \mathrm{~mm}$ relative to an anatomic landmark or any migration leading to symptoms or requiring intervention. Typical landmarks for comparison and assessment of migration are the superior mesenteric artery (SMA) and renal arteries. Migration can be asymptomatic, or can result in type I endoleak and a subsequent rupture. Stent graft migration can also result in branch vessel occlusion, usually renal arteries and internal iliac arteries. Major device migration increases the risk of intragraft thrombosis.

Graft kinking occurs in about 3\% of patients after EVAR, more commonly after device distal migration with an intact distal attachments site [16]. Kinking can lead to type I and type III endoleak and is strongly associated with limb thrombosis due to turbulent flow in the kinked stent graft [17]. Stent graft thrombosis occurs usually in the first 2 months following surgery [18]. Frequency of stent-graft limb thrombosis strongly depends on the type of graft and ranges from $5 \%$ to $40 \%$ depending on the device type [17, $19,20]$. 
Graft infection is a rare complication of EVAR, occurring in less than $1 \%$ of patients, but is related to high mortality rates of $6-11 \%[21,22]$. Graft infection can lead to periaortic abscesses, aorto-enteric fistulas, and systemic sepsis [23]. Air within the aortic sac, mesenteric inflammation adjacent to the stent graft, perigraft fluid collections, and thrombus enhancement are imaging symptoms of graft infection in computed tomography (CT).

Access site complications include local wound complications and access artery injuries. Groin hematoma, infection, and lymphocele occur with a frequency of 1-10\% [24]. They are usually diagnosed with ultrasound and CT is required only in advanced cases. Significant complications, including iliac artery dissection, thrombosis and pseudoaneurysm formation, occur in up to 3\% and require CT imaging [17].

\section{Imaging methods}

Imaging methods used in the post-EVAR surveillance include plain X-ray, ultrasound, computed tomography, magnetic resonance imaging, and conventional angiography. It seems important to understand the unique advantages and disadvantages of each modality to personalize diagnostics for optimal patient outcome.

\section{Radiography}

Plain radiography of the abdomen performed in anteroposterior and lateral projections is useful in the assessment of the graft position and integrity (Photo 1). Therefore, prosthesis migration, separation of modules, and wire fractures may be easily detected using radiography [25]. As the technique does not suffer from artifacts related to metallic objects, it can be even more sensitive to mechanical damage than cross-sectional modalities [13]. Obviously, plain radiographs do not allow for imaging of the aneurysm size and leaks so they cannot be used as a standalone method of follow-up [25].

\section{Ultrasound}

Conventional ultrasound (US) with Doppler option and contrast-enhanced ultrasound (CEUS) are less invasive but are considered less accurate than CT. However, in many centers US is the primary imaging method after EVAR (Photo 2). The US is a non-invasive and low-cost alternative to other di-

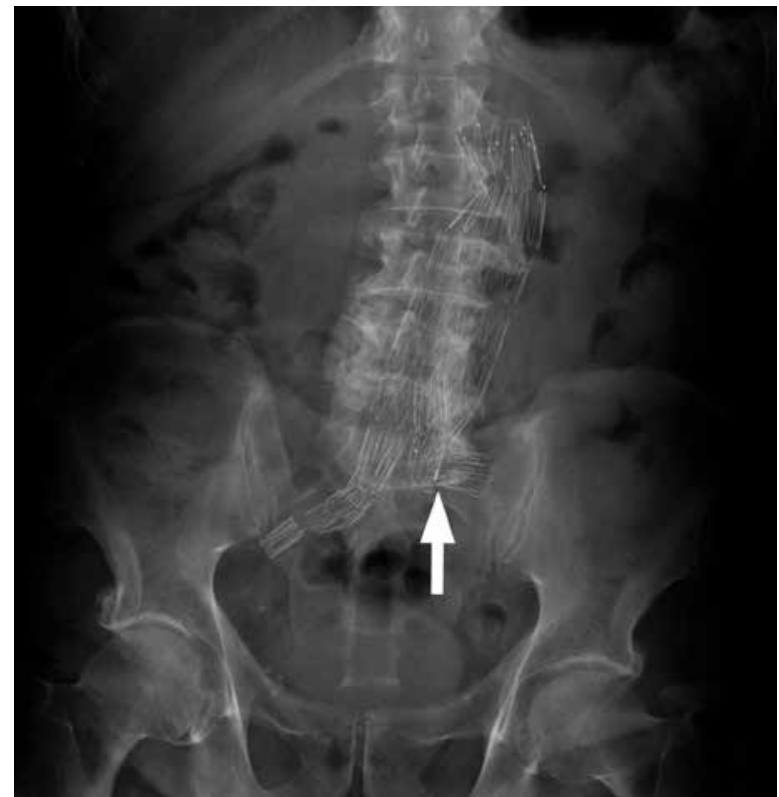

Photo 1. Plain abdominal radiograph presenting kinking of the left iliac arm of the graft (arrow)

agnostic imaging modalities. The undeniable advantages of US are non-invasiveness, wide availability, low cost, and lack of ionizing radiation. The ability of Doppler US to identify flow direction in endoleaks is a great advantage over computed tomography angiography (CTA). Major disadvantages of US remain operator skill and technique dependency, equipment dependency and attenuation artifacts in large body habitus patients. There is still no consensus among researchers on the value of US in identifying the primary complication of EVAR, i.e. endoleak. According to various authors, the sensitivity of US in this regard ranges from $12 \%$ to $100 \%$ [26-31]. Those numbers

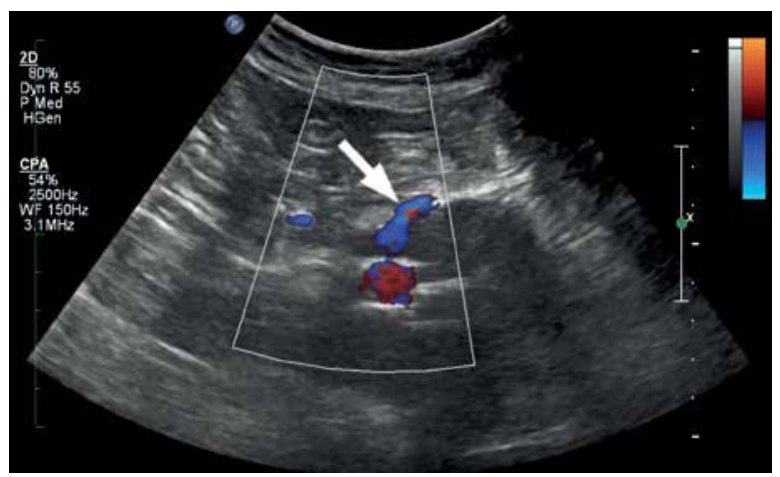

Photo 2. Patient presented in Photo 1. Doppler ultrasound shows an endoleak type $\mathrm{Ib}$ due to inaccurate connection between the main graft body and the left iliac extension 
underline the significant subjectivity of sonography and may be due to the different experience of observers, differences in ultrasound equipment quality, and the wide range of patients enrolled in studies (20-561 patients) [32].

On the other hand, CEUS is nowadays proposed as a routine diagnostic modality for follow-up after EVAR and should be extended by CT only when the ultrasound is positive [33]. Based on their recent meta-analysis that included 42 primary studies with 4220 participants, Abraha et al. calculated summary estimates in detecting endoleaks. US showed 0.72 sensitivity and 0.95 specificity whereas respective summary estimates for CEUS were 0.91 and 0.89 . The CEUS is a technique using intravenous administered contrast agent with stabilized microspheres consisting of sulfur hexafluoride or perfluorocarbon encapsulated by a phospholipid shell. Microspheres do not affect renal function and are released via the respiratory tract. As the contrast medium has a very good safety profile, this modality can be used particularly in patients with contraindications to iodine and gadolinium-based contrast media that are used in CTA and magnetic resonance angiography (MRA). The CEUS presents detailed vascular morphology and enables real-time quantification of blood flow [34], which in some studies resulted in a higher specificity than that of CTA in endoleak classification [35]. In a recent retrospective study by Chisci et al. The CEUS was used as a reference method of follow-up [36]. In their material 318 CEUS examinations were performed with no procedure-related complications and allowed for $90 \%$ reduction in the number of CTAs, thereby reducing radiation exposure for patients.

\section{Computed tomography angiography}

The CTA is the current reference standard and a workhorse for surveillance after $\operatorname{EVAR}[13,37]$. The CTA is widely available, less operator-dependent than US, and can be performed rapidly in unstable patients [37]. The superb spatial resolution of CTA enables precise measurement of aortic diameters and detection of both mechanical (stent graft kinking, migration, fracture) and soft tissue complications (inflammation, abscess formation, new aneurysms formation). However, due to inability to assess blood flow direction, CTA may present some limitations in endoleak classification [38].
Repeated follow-up CTAs in a patient after EVAR are related to a significant risk of contrast-induced acute kidney injury (CI-AKI) [39] and potentially carcinogenic cumulative radiation dose [40]. Patients after EVAR due to usually advanced age and frequent atherosclerosis present an increased risk for CI-AKI. Interestingly, we could not find any study published on kidney injury prevalence in this group. On the other hand, Maaniitty et al. found CI-AKI in 3.5\% of in patients undergoing coronary CTA and permanent kidney injury in $0.2 \%$ [41]. In our opinion, these low numbers reflect contemporary common awareness of nephrotoxic properties of contrast media and widespread knowledge on the role of hydration before CTA [39]. The harmful effect of a radiation dose is much more difficult to assess due to the stochastic nature of carcinogenesis. Nyheim et al. examined the impact of age and follow-up regime over time on cumulative radiation exposure and attributable cancer risk after EVAR [40]. They suggested using a simplified surveillance protocol in younger patients as attributable cancer risk due to a 15-year follow-up was $0.65 \%$. Bobadilla et al. proposed using a single unenhanced phase with real-time aneurysm sac volume calculation. In the case of significant (> $2 \%$ ) enlargement, further arterial and delayed phases are performed [42]. Another way to reduce radiation exposure is skipping the arterial phase when the main indication for imaging is endoleak suspicion [43].

Also dual-energy CT (DECT) is a promising technique in this regard, as it is able to retrospectively produce a so-called virtual unenhanced phase scanning the patient once, after contrast media administration. Therefore, DECT scanning results in $28-42 \%$ mean dose reduction with comparable diagnostic accuracy compared to biphasic single energy CTA [44, 45]. In another study, the effective dose reduction in DECT was $62 \%$ DECT compared to conventional triple-phase CTA with same diagnostic accuracy [46]. Another way of decreasing radiation exposure can be lowering the tube voltage from 120 to $80 \mathrm{kVp}$ or even $80 \mathrm{kVp}$. In the study by Wintersperger et al. reduced tube voltage resulted in a mean dose reduction of $34 \%$ [47]. An advantage of low kVp imaging is increased image contrast, which allows for contrast medium dose reduction. A drawback is increased image noise that degrades overall image readability. A solution would be applying iterative or model-based image reconstructions [48]. These techniques use multiple advanced mathematical 
operations to optimize the image by reducing the noise. Iterative reconstructions are widely available in contemporary CT scanners and should be used routinely. Model-based reconstructions seem to be still a work-in-progress since the technology is expensive and time-consuming. However, it enables a $73 \%$ dose reduction as compared with low-dose conventional adaptive iterative reconstruction while maintaining diagnostic accuracy [49].

The diagnostic accuracy of CTA for detection of endoleaks is higher than that of digital subtraction angiography (DSA) and Doppler US [38]. However, one has to keep in mind that detection of endoleaks is strongly dependent on the scanning protocol. A traditional acquisition consists of an unenhanced phase, arterial phase, and 120-s delayed phase. The unenhanced phase allows for differentiation between endoleak and calcifications or embolizing material. The arterial phase allows for a detailed presentation of the aortic lumen and aortic branches as well as high-flow endoleaks. The delayed phase is aimed at visualization of slow-flow endoleaks (Photo 3). Some authors suggested using a 300-s delayed phase to present very low-flow endoleaks $[50,51]$. The Eurostar study proposed performing only one delayed-phase CT with 3-mm slice thickness [52]. From our personal perspective three phases and thin reconstruction slices are necessary for reliable post-EVAR surveillance. A basic scanning protocol is presented in Table I. However, we strongly suggest tailoring the parameters to reduce the radiation dose according to the scanner capability.

The primary aim of follow-up is aneurysm size assessment as persistent growth after EVAR is a major risk factor of rupture. It is generally assumed that the size reduction indicates the successful functioning of the prosthesis but data on this subject remain inconclusive [53]. The most accurate assessment of the aneurysm can be obtained by evaluation of its volume. However, significant discrepancies between the assessment of maximal dimensions of the aneu-
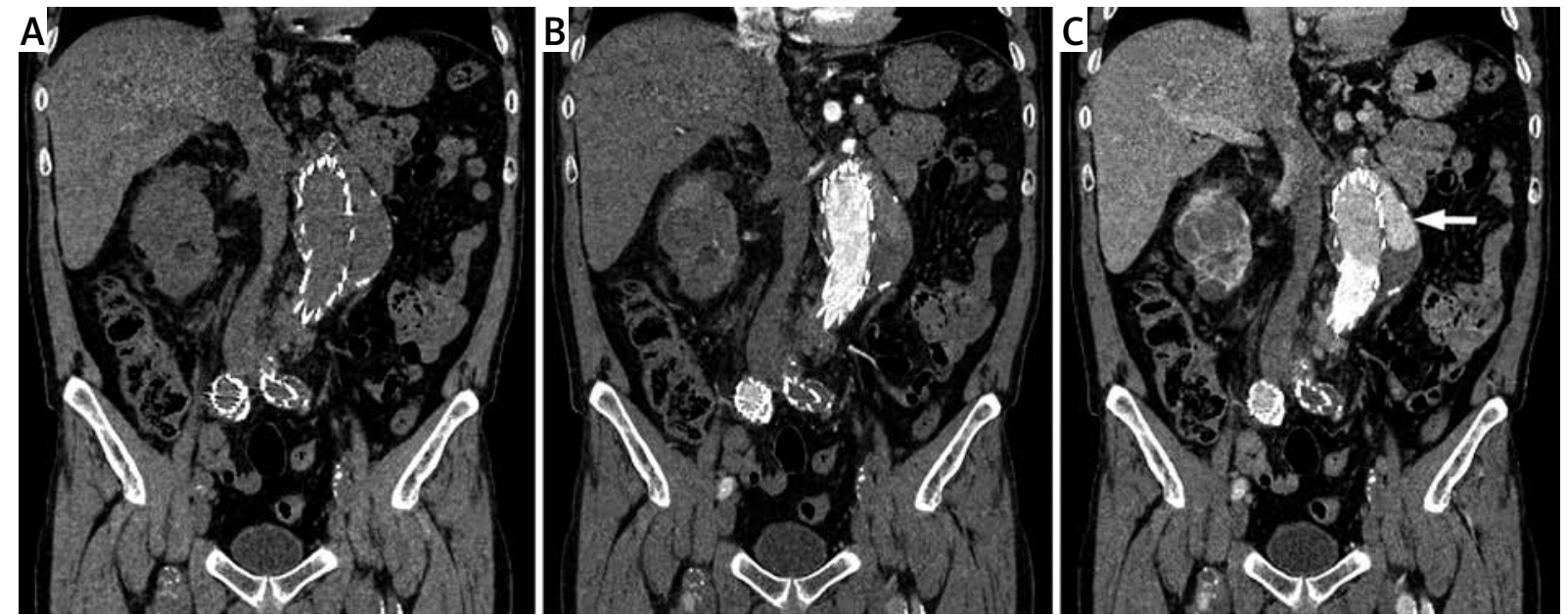

Photo 3. The CT in the post-EVAR surveillance in non-enhanced phase (A), 30-s arterial phase (B), and delayed 120-s phase (C). A type la endoleak is visible only in the delayed phase (arrow)

Table I. A basic CTA protocol for post-EVAR follow-up

\begin{tabular}{|c|c|c|}
\hline Parameter & Recommendations & Notes \\
\hline Coverage & From diaphragm to lower surface of pubic symphysis & \\
\hline Phases & Non-enhanced, arterial, delayed (60 s) & $\begin{array}{l}\text { Non-enhanced phase may be skipped if DECT } \\
\text { is used }\end{array}$ \\
\hline Scan parameters & $\begin{array}{c}\text { Slice } 1 \mathrm{~mm} \text {, pitch } 1 \mathrm{~mm}, 120 \mathrm{kVp} \text {, automatic mAs } \\
\text { adjustment }\end{array}$ & $\begin{array}{l}\text { Available dose reduction techniques strongly } \\
\text { recommended }\end{array}$ \\
\hline Contrast media & $70 \mathrm{ml}+30 \mathrm{ml} \mathrm{NaCl}$ at $4 \mathrm{ml} / \mathrm{s}$ & $\geq 350 \mathrm{mg} \mathrm{l} / \mathrm{ml}$ \\
\hline Bolus tracking & Aorta at level of diaphragm & Threshold 120 HU, 8-s delay \\
\hline
\end{tabular}


rysm and changes in its volume have been observed $[54,55]$. In order to maintain adequate intraobserver and intraobserver reproducibility of measurements, lumen centerline curved planar reconstructions or at least 3D multiplanar reformations of image datasets are strongly recommended [56]. The CTA also enables a number of other image reconstruction possibilities including volume rendering (VR), shaded surface display (SSD) and virtual endoscopy (VE). In our opinion they do not increase the diagnostic accuracy of tomography but may be useful for initial recognition of aneurysm anatomy and as a tool for clinico-radiological consultations.

An important problem in post-EVAR surveillance using CTA is the presence of stent-related beam-hardening artifacts (Photo 4). Similar artifacts may be produced by coils, Lipiodol used as a component of embolic fluid, and the tantalum component of Onyx that are used for endoleak embolization. All these artifacts may obscure small leaks of contrast medium, but measurement of aneurysm sac size is still adequate [57]. DECT seemed to be a solution since it offers a projection-based iterative postprocessing method to reduce photon starvation, beam hardening and streak artifacts caused by metallic implants. However, despite better stent-graft visualization, recent studies have shown that this technique reduces small endoleak visualization [58].

\section{Magnetic resonance angiography}

Magnetic resonance angiography is a good alternative for post-EVAR follow-up, especially in patients with contraindications to iodinated contrast agents that are used in CTA. Another advantage of MRA is the lack of exposure to ionizing radiation. Limitations of MRA include its limited availability, high costs, and long scan time. Common contraindications to MRI include ferromagnetic implants and foreign bodies, electronic implants, and claustrophobia. However, in the case of implanted pacemakers or cardioverters/ defibrillators it is worth considering the examination as modern devices are either MRI-compatible or MRI-conditionally compatible and successful scanning can be performed under specific conditions.

In patients with chronic kidney disease administration of gadolinium contrast media is related to risk of nephrogenic systemic fibrosis (NSF). In such patients, non-contrast enhanced MRA can be performed using steady state free precession
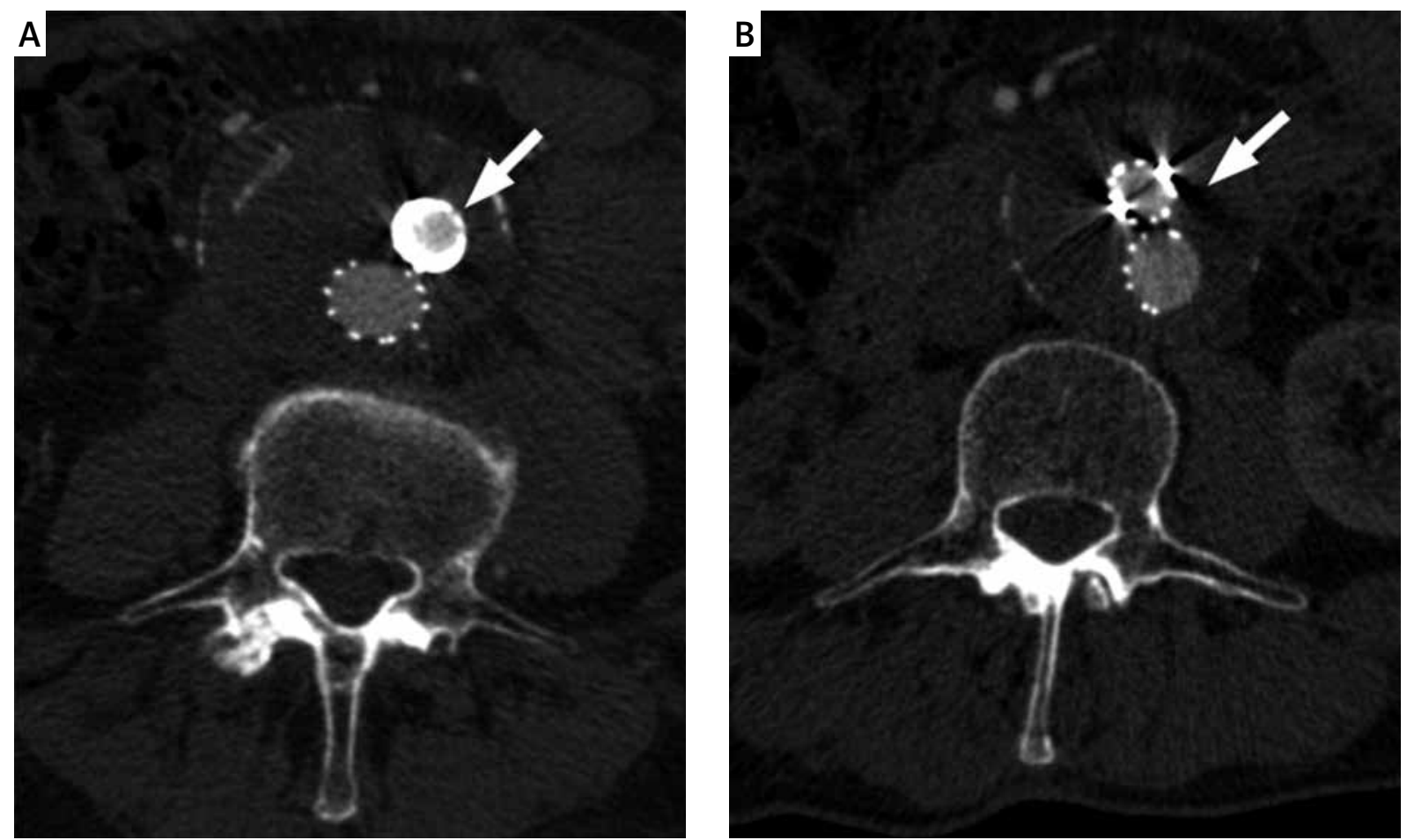

Photo 4. The CT in the post-EVAR surveillance. Stent-related blooming artifacts (arrow) hamper artery lumen evaluation (A). Photon-starving artifacts (arrow) may influence endoleak detection (B) 
T2-weighted sequences (e.g. FIESTA, TrueFISP, or Balanced-FFE) but at the cost of lower diagnostic accuracy [59]. Another potential limitation related to contrast media is recently discussed gadolinium retention in the globus pallidus, dentate nucleus, thalamus and pons $[60,61]$. The retention is clearly related to MRI contrast media administration and is proportional to the cumulative gadolinium dose, which is important in long-term follow-up that requires repeated examinations. On the other hand, pathological significance of this phenomenon remains unknown. In a microscopic study no scarring or gliosis in the territory of accumulation was found [61]. Moreover, to date no clinical manifestations of gadolinium retention have been found.

The efficiency of MRA strongly depends on the graft material because metal-induced susceptibility artifacts can significantly reduce image quality (Photo 5). Nitinol endografts are most suitable for MRA surveillance, since Nitinol does not produce such artifacts. Also platinum coils used for endoleak embolization do not preclude MRA assessment [62]. Conversely, nickel-alloy and stainless-steel components should not be examined using MRA [63]. In patients with such devices the preferred imaging modality should be CTA or CEUS. We strongly recommend verification of the graft MRI compatibility using either the implant documentation or web bases of knowledge (e.g. http://mrisafety.com).

The most commonly used post-EVAR surveillance protocol consists of T1-weighted acquisition before and after contrast medium application in arterial and late phases. The arterial phase presents the aortic lumen and the late phase is recommended for endoleak detection [64]. A recent systematic review showed that MRI was more sensitive than CTA in detection of endoleaks, especially of type II [65]. In a group of 369 patients, 146 endoleaks were detected by CTA. The MRI detected all but two and also found 132 additional endoleaks compared to CTA. Therefore, MRI should be considered in patients with continued AAA growth and negative or uncertain findings in CTA. Detection of type II endoleaks is important due to the fact that with the increase in size of the aneurysm, they require immediate treatment [13].

Time-resolved MRA is a modification of the basic sequence in which high temporal resolution enables assessment of blood flow dynamics but at the cost of reduced spatial resolution and increased susceptibility artifacts $[66,67]$. As a dynamic modality, it can be an alternative to invasive angiography in differentiation of the endoleak types $[68,69]$. However, inferior spatial resolution may result in limited sensitivity to small leaks. Another method that increases sensitivity of MRA to endoleaks is the use of blood-pool contrast agents, which present prolonged intravascular retention [70]. Blood-pool media bind to albumin and have a blood half-life of approximately $15 \mathrm{~h}$. They also have very high relaxivity. Therefore they have the potential to detect low-flow endoleaks that may be occult on CTA [70]. Low availability of blood-pool contrast media and advanced technical requirements have limited
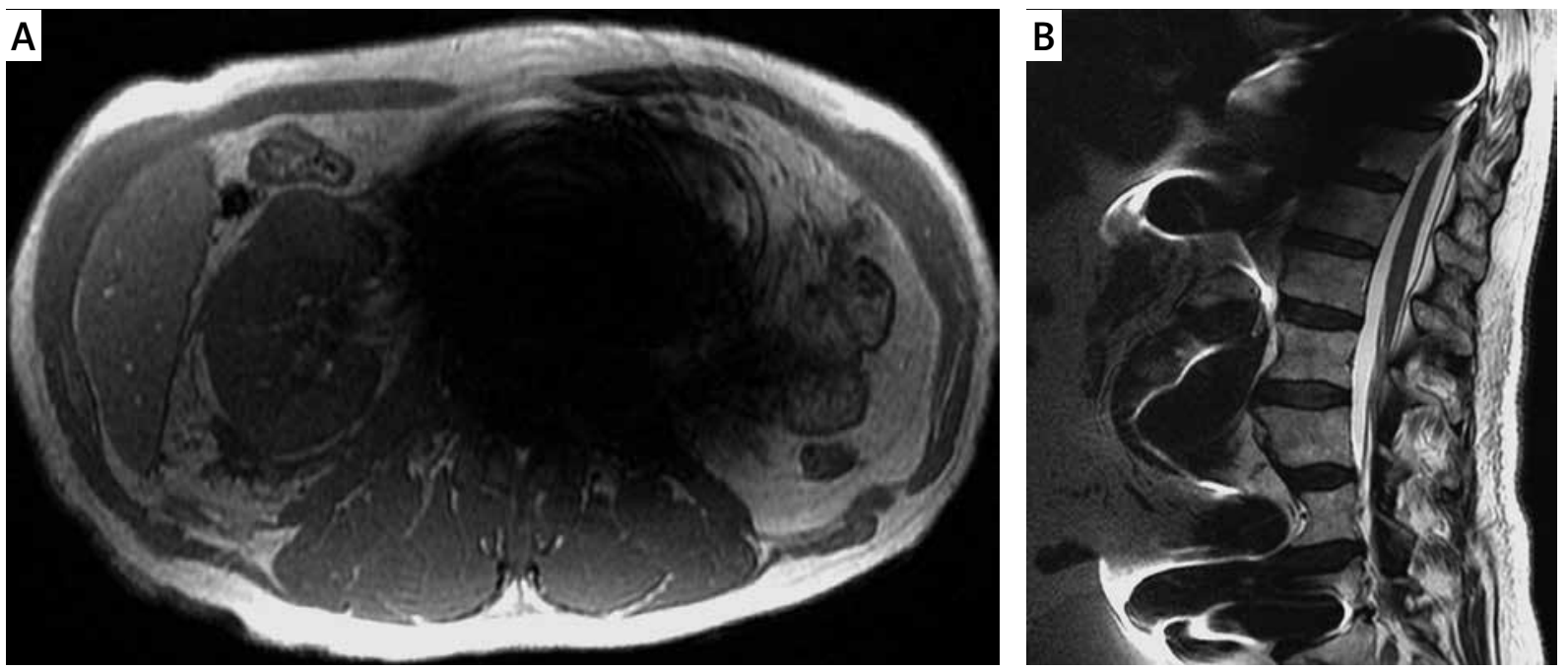

Photo 5. Non-contrast enhanced MRI. Strong susceptibility artifacts produced by an aortic Zenith graft on axial (A) and sagittal (B) projection 
the use of this technique so far. Recently, four-dimensional non-contrast enhanced time-resolved phase contrast MRA was proposed for post-EVAR surveillance [71]. This technique is able to precisely present the bloodstream in the aorta and to analyze different hemodynamic and biomechanical forces acting on the intermodular connections of a multi-component stent graft using computational modeling [72]. As a dynamic modality, phase contrast MRA can be used for both the detection of endoleaks and the differentiation between endoleak types. Additionally it can show pathological, turbulent flow dynamics that predispose to thrombosis and endoleak [73, 74].

Despite its high sensitivity and specificity in endoleak detection, MRA has not yet become a routine method of post-EVAR surveillance. Apart from the above-mentioned common limitations of MRI, it seems that the challenging scanning protocol and the lack of expertise outside of major centers are the most important factors that restrict widespread use of MRA. On the other hand, MRI should be used to diagnose medullary ischemia, which may be a complication of EVAR [75].

\section{Digital subtraction angiography}

The DSA is not recommended for the routine postEVAR follow-up [7]. The main disadvantage of this modality is its invasiveness related to the artery puncture, the possibility of the vessel dissection or plaque mobilization, the significant radiation dose, and the intraarterial contrast medium application [76].

Compared to CTA, DSA presents significantly lower sensitivity in detecting endoleaks (92\% vs. 63\%, respectively) [77]. However, due to the dynamic nature of DSA, it allows for the assessment of blood flow direction and therefore it may be helpful in proper endoleak classification in selected cases. In CTA, contrast medium in lumbar arteries and inferior mesenteric arteries may result in problems in distinguishing between types I or III and type II. In a study by Stavropoulos et al. it was found that the use of DSA after CTA resulted in a significant change of diagnosed endoleak type and a change in treatment in $11 \%$ of patients [38].

\section{Follow-up recommendations}

Complications occurring in patients after EVAR are well documented, and the need for systematic surveillance seems to be clear [78]. Recommenda- tions by the European Society for Vascular Surgery (ESVS) suggest that all patients should have computed tomography angiography (CTA) and plain radiographs with anteroposterior and lateral projections at 30 days after the procedure [13]. If there is any endoleak or less than one stent component or iliac overlap, CTA at 6 months and 12 months with plain radiographs should be done with adequate treatment if indicated. In patients with no early endoleak and good component overlap, the routine 6-month CTA could be omitted, but CTA and plain radiographs should be done after 1 year. At 12 months, if there is no endoleak and a stable or shrinking AAA, yearly Doppler US is recommended with plain radiographs using a standardized protocol with antero-posterior and lateral projections to assess device migration, stent fractures and modular disconnections. If the patient's body habitus precludes adequate US, then non-contrast CT with plain radiographs can be substituted. Any increasing aneurysm diameter or new endoleak, after prior negative imaging, should prompt complete imaging with CTA and plain radiographs.

However, it is worth mentioning that the presented recommendations are now 7 years old. Since their publication both stent graft quality and imaging techniques have improved significantly. Therefore, we believe there is a need for an update of guidelines with a reduced role of plain radiograms and increased application of US, including CEUS. On the other hand, recently Garg et al. suggested that less frequent imaging than suggested by ESVS guidelines was not associated with worse outcomes [79]. Other studies indicate that only up to $9 \%$ of patients actually benefit from surveillance imaging, as the imaging alone initiated secondary interventions in such a proportion of cases. That implies that more than $90 \%$ of post-EVAR patients received no benefits from follow-up [80, 81]. On the other hand, Leurs et al. reported that initially high-risk patients, despite more intensive imaging surveillance, had still more complications and higher mortality after EVAR [52]. Thus, individually scheduled follow-up instead of strict adherence to recommendations should be considered.

\section{Personal perspective}

In clinical routine, CTA without a doubt remains the first line modality in post-EVAR surveillance because of its precision in diagnostics and availability. 
However, apart from DSA, CTA seems to be the most invasive modality due to exposure to radiation and potentially nephrotoxic contrast media. Therefore, in the time of personalized medicine, we should tailor diagnostic schedules both to receive accurate diagnosis and to reduce risk of complications.

Despite current recommendations of ESVS and its relative non-invasiveness, we should skip plain radiograph as it presents very limited diagnostic accuracy regarding the most clinically significant complications. From our personal perspective, Doppler US is an excellent tool for late follow-up in patients with no complications in the previous two or three CTAs. Such subjects present low probability of further stent-graft-related pathologies. On the other hand, significant endoleaks, which are worth treatment, are easily visible on US. The CEUS is a method with a special potential for post-EVAR surveillance. It presents sensitivity and specificity to endoleaks that are similar to CTA and an excellent safety profile. However, it requires high experience of the operator. Therefore, CEUS is an ideal modality for tertiary referral centers with high loads of patients. Finally, it is worth underlining the importance of clinico-radiological consultations, which are an essential part of contemporary patient management.

\section{Conclusions}

Surveillance imaging is an important part of patient management after EVAR and CTA is the current first choice modality for follow-up. However, since current imaging recommendations seem not to adhere to current imaging possibilities, a more personalized approach is necessary with special attention paid to side effects of CTA and cost effectiveness.

\section{Conflict of interest}

The authors declare no conflict of interest.

\section{References}

1. Aggarwal S, Qamar A, Sharma V, et al. Abdominal aortic aneurysm: a comprehensive review. Exp Clin Cardiol 2011; 16: 11-5.

2. Pearce WH, Zarins CK, Bacharach JM; American Heart Asso ciation Writing Group 6. Atherosclerotic peripheral vascular disease symposium II: controversies in abdominal aortic aneurysm repair. Circulation 2006; 118: 2860-3.

3. Jongkind V, Yeung KK, Akkersdijk GJ, et al. Juxtarenal aortic aneurysm repair. J Vasc Surg 2010; 52: 760-7.

4. Vardulaki KA, Walker NM, Couto E, et al. Late results concerning feasibility and compliance from a randomized trial of ultraso- nographic screening for abdominal aortic aneurysm. Br J Surg 2002; 89: 861-4.

5. Scott RA, Bridgewater SG, Ashton HA. Randomized clinical trial of screening for abdominal aortic aneurysm in women. $\mathrm{Br}$ J Surg 2002; 89: 283-5.

6. Alcorn HG, Wolfson SK Jr, Sutton-Tyrrell K, et al. Risk factors for abdominal aortic aneurysms in older adults enrolled in The Cardiovascular Health Study. Arterioscler Thromb Vasc Biol 1996; 16: 963-70.

7. Brewster DC, Cronenwett JL, Hallett JW Jr, et al. Joint Council of the American Association for Vascular Surgery and Society for Vascular Surgery. Guidelines for the treatment of abdominal aortic aneurysms. Report of a subcommittee of the Joint Council of the American Association for Vascular Surgery and Society for Vascular Surgery. J Vasc Surg 2003; 37: 1106-17.

8. Brown LC, Powell JT, Thompson SG, et al. The UK EndoVascular Aneurysm Repair (EVAR) trials: randomised trials of EVAR versus standard therapy. Health Technol Assess 2012; 16: 1-218.

9. Volodos NL, Karpovich IP, Shekhanin VE, et al. [A case of distant transfemoral endoprosthesis of the thoracic artery using a self-fixing synthetic prosthesis in traumatic aneurysm]. Grudn Khir 1988; 6: 84-6.

10. Parodi JC, Palmaz JC, Barone HD. Transfemoral intraluminal graft implantation for abdominal aortic aneurysms. Ann Vasc Surg 1991; 5: 491-9.

11. d'Audiffret A, Desgranges P, Kobeiter DH, et al. Follow-up evaluation of endoluminally treated abdominal aortic aneurysms with duplex ultrasonography: validation with computed tomography. J Vasc Surg 2001; 33: 42-50.

12. White GH, Yu W, May J. Endoleak - a proposed new terminology to describe incomplete aneurysm exclusion by an endoluminal graft. J Endovasc Surg 1996; 3: 124-5.

13. Moll FL, Powell JT, Fraedrich G, et al.; European Society for Vascular Surgery. Management of abdominal aortic aneurysms clinical practice guidelines of the European society for vascular surgery. Eur J Vasc Endovasc Surg 2011; 41 Suppl. 1: S1-58.

14. Cao P, De Rango P, Verzini F, et al. Endoleak after endovascular aortic repair: classification, diagnosis and management following endovascular thoracic and abdominal aortic repair. J Cardiovasc Surg 2010; 51: 53-69.

15. Carpenter JP, Anderson WN, Brewster DC, et al.; Lifepath Investigators. Multicenter pivotal trial results of the Lifepath System for endovascular aortic aneurysm repair. J Vasc Surg 2004; 39: 34-43.

16. Carroccio A, Faries PL, Morrissey NJ, et al. Predicting iliac limb occlusions after bifurcated aortic stent grafting: anatomic and device-related causes. J Vasc Surg 2002; 36: 679-84.

17. Maleux G, Koolen M, Heye S, et al. Limb occlusion after endovascular repair of abdominal aortic aneurysms with supported endografts. J Vasc Interv Radiol 2008; 19: 1409-12.

18. Maldonado TS, Rockman CB, Riles E, et al. Ischemic complications after endovascular abdominal aortic aneurysm repair. J Vasc Surg 2004; 40: 703-9.

19. Amesur NB, Zajko AB, Orons PD, et al. Endovascular treatment of iliac limb stenoses or occlusions in 31 patients treated with the ancure endograft. J Vasc Interv Radiol 2000; 11: 421-8.

20. Fairman RM, Baum RA, Carpenter JP, et al.; Phase II EVT Investigators. Limb interventions in patients undergoing treatment 
with an unsupported bifurcated aortic endograft system: a review of the Phase II EVT Trial. J Vasc Surg 2002; 36: 118-26.

21. Ducasse E, Calisti A, Speziale F, et al. Aortoiliac stent graft infection: current problems and management. Ann Vasc Surg 2004 18: 521-6.

22. Fatima J, Duncan AA, de Grandis E, et al. Treatment strategies and outcomes in patients with infected aortic endografts. J Vasc Surg 2013; 58: 371-9.

23. Sharif MA, Lee B, Lau LL, et al. Prosthetic stent graft infection after endovascular abdominal aortic aneurysm repair. I Vasc Surg 2007; 46: 442-8.

24. Liaw JV, Clark M, Gibbs R, et al. Update: complications and management of infrarenal EVAR. Eur J Radiol 2009; 71: 541-51.

25. Murphy M, Hodgson R, Harris PL, et al. Plain radiographic surveillance of abdominal aortic stent-grafts: the Liverpool/Perth protocol. J Endovasc Ther 2003; 10: 911-2.

26. McWilliams RG, Martin J, White D, et al. Detection of endoleak with enhanced ultrasound imaging: comparison with biphasic computed tomography. J Endovasc Ther 2002; 9: 170-9.

27. Thompson MM, Boyle JR, Hartshorn T, et al. Comparison of computed tomography and duplex imaging in assessing aortic morphology following endovascular aneurysm repair. Br I Surg 1998; 85: 346-50.

28. Fletcher J, Saker K, Batiste P, et al. Colour Doppler diagnosis of perigraft flow following endovascular repair of abdominal aortic aneurysm. Int Angiol 2000; 19: 326-30.

29. Parent FN, Meier GH, Godziachvili V, et al. The incidence and natural history of type I and II endoleak: a 5-year follow-up assessment with color duplex ultrasound scan. J Vasc Surg 2002; 35: 474-81.

30. McLafferty RB, McCrary BS, Mattos MA, et al. The use of colorflow duplex scan for the detection of endoleaks. I Vasc Surg 2002; 36: 100-4.

31. Collins JT, Boros MJ, Combs K. Ultrasound surveillance of endovascular aneurysm repair: a safe modality versus computed tomography. Ann Vasc Surg 2007; 21: 671-5.

32. Karanikola E, Dalainas I, Karaolanis G, et al. Duplex ultrasound versus computed tomography for the postoperative follow-up of endovascular abdominal aortic aneurysm repair. Where do we stand now? Int J Angiol 2014; 23: 155-64.

33. Abraha I, Luchetta ML, De Florio R, et al. Ultrasonography for endoleak detection after endoluminal abdominal aortic aneurysm repair. Cochrane Database Syst Rev 2017; 6: CD010296.

34. Białecki M, Białecka A, Laskowska K, et al. Contrast-enhanced ultrasonography for the determination of Crohn's disease activity - preliminary experience. Pol J Radiol 2014; 79: 70-4.

35. Carrafiello G, Recaldini C, Lagan D, et al. Endoleak detection and classification after endovascular treatment of abdominal aortic aneurysm: value of CEUS over CTA. Abdom Imaging 2008; 33: 357-62.

36. Chisci E, Harris L, Guidotti A, et al. Endovascular aortic repair follow up protocol based on contrast enhanced ultrasound is safe and effective. Eur J Vasc Endovasc Surg 2018; 56: 40-7.

37. Hallett RL, Ullery BW, Fleischmann D. Abdominal aortic aneurysms: pre- and post-procedural imaging. Abdom Radiol 2018; 43: 1044-66.
38. Stavropoulos SW, Clark TW, Carpenter JP, et al. Use of CT angiography to classify endoleaks after endovascular repair of abdominal aortic aneurysms. J Vasc Interv Radiol 2005; 16: 663-7.

39. Serafin Z, Karolkiewicz M, Gruszka M, et al. High incidence of nephropathy in neurosurgical patients after intra-arterial administration of low-osmolar and iso-osmolar contrast media. Acta Radiol 2011; 52: 422-9

40. Nyheim T, Staxrud LE, Jørgensen JJ, et al. Radiation exposure in patients treated with endovascular aneurysm repair: what is the risk of cancer, and can we justify treating younger patients? Acta Radiol 2017; 58: 323-30.

41. Maaniitty T, Stenström I, Uusitalo V, et al. Incidence of persistent renal dysfunction after contrast enhanced coronary CT angiography in patients with suspected coronary artery disease. Int I Cardiovasc Imaging 2016; 32: 1567-75.

42. Bobadilla JL, Suwanabol PA, Reeder SB, et al. Clinical implications of non-contrast-enhanced computed tomography for follow-up after endovascular abdominal aortic aneurysm repair. Ann Vasc Surg 2013; 27: 1042-8.

43. Macari M, Chandarana H, Schmidt B, et al. Abdominal aortic aneurysm: can the arterial phase at $\mathrm{CT}$ evaluation after endovascular repair be eliminated to reduce radiation dose? Radiology 2006; 241: 908-14.

44. Ascenti G, Mazziotti S, Lamberto S, et al. Dual-energy CT for detection of endoleaks after endovascular abdominal aneurysm repair: usefulness of colored iodine overlay. AJR Am J Roentgenol 2011; 196: 1408-14.

45. Javor D, Wressnegger A, Unterhumer S, et al. Endoleak detection using single-acquisition split-bolus dual-energy computer tomography (DECT). Eur Radiol 2017; 27: 1622-30.

46. Buffa V, Solazzo A, D’Auria V, et al. Dual-source dual-energy CT: dose reduction after endovascular abdominal aortic aneurysm repair. Radiol Med 2014; 119: 934-41.

47. Wintersperger B, Jakobs T, Herzog P, et al. Aorto-iliac multidetector-row CT angiography with low KV settings: improved vessel enhancement and simultaneous reduction of radiation dose. Eur Radiol 2005; 15: 334-41.

48. Böning G, Rotzinger RA, Kahn JF, et al. Tailored CT angiography in follow-up after endovascular aneurysm repair (EVAR): combined dose reduction techniques. Acta Radiol 2018: 284185118756952; doi: 10.1177/0284185118756952.

49. Hansen NJ, Kaza RK, Maturen KE, et al. Evaluation of low-dose CT angiography with model-based iterative reconstruction after endovascular aneurysm repair of a thoracic or abdominal aortic aneurysm. AJR Am J Roentgenol 2014; 202: 648-55.

50. Rozenblit AM, Patlas M, Rosenbaum AT, et al. Detection of endoleaks after endovascular repair of abdominal aortic aneurysm: value of unenhanced and delayed helical CT acquisitions. Radiology 2003; 227: 426-33.

51. lezzi R, Cotroneo AR, Filippone A, et al. Multidetector CT in abdominal aortic aneurysm treated with endovascular repair: are unenhanced and delayed phase enhanced images effective for endoleak detection? Radiology 2006; 241: 915-21.

52. Leurs LJ, Laheij RJ, Buth J; EUROSTAR Collaborators. What determines and are the consequences of surveillance intensity af- 
ter endovascular abdominal aortic aneurysm repair? Ann Vasc Surg 2005; 19: 868-875.

53. Nowicka M, Kowalczyk A, Rusak G, et al. Evaluation the aortic aneurysm remodeling after a successful stentgraft implantation. Pol J Radiol 2016; 81: 486-90.

54. Kritpracha B, Beebe HG, Comerota AJ. Aortic diameter is an insensitive measurement of early aneurysm expansion after endografting. J Endovasc Ther 2004; 11: 184-90.

55. Bargellini I, Cioni R, Petruzzi P, et al. Endovascular repair of abdominal aortic aneurysms: analysis of aneurysm volumetric changes at mid-term follow-up. Cardiovasc Intervent Radiol 2005; 28: 426-33.

56. Pandey N, Litt HI. Surveillance imaging following endovascular aneurysm repair. Semin Intervent Radiol 2015; 32: 239-48.

57. Stavropoulos SW, Marin H, Fairman RM, et al. Recurrent endoleak detection and measurement of aneurysm size with CTA after coil embolization of endoleaks. J Vasc Interv Radiol 2005; 16: 1313-7.

58. Boos J, Fang J, Heidinger BH, et al. Dual energy CT angiography: pros and cons of dual-energy metal artifact reduction algorithm in patients after endovascular aortic repair. Abdom Radiol 2017; 42: 749-58.

59. Resta EC, Secchi F, Giardino A, et al. Non-contrast MR imaging for detecting endoleak after abdominal endovascular aortic repair. Int J Cardiovasc Imaging 2013; 29: 229-35.

60. Kanda T, Ishii K, Kawaguchi H, et al. High signal intensity in the dentate nucleus and globus pallidus on unenhanced T1-weighted MR images: relationship with increasing cumulative dose of a gadolinium-based contrast material. Radiology 2014; 270: 834-41.

61. McDonald RJ, McDonald JS, Kallmes DF, et al. Intracranial gadolinium deposition after contrast-enhanced MR imaging. Radiology 2015; 275: 772-82.

62. Ayuso JR, de Caralt TM, Pages M, et al. MRA is useful as a follow-up technique after endovascular repair of aortic aneurysms with nitinol endoprostheses. J Magn Reson Imaging 2004; 20: 803-10.

63. van der Laan MJ, Bartels LW, Bakker CJ, et al. Suitability of 7 aortic stent-graft models for MRI-based surveillance. J Endovasc Ther 2004; 11: 366-71.

64. Alerci M, Oberson M, Fogliata A, et al. Prospective, intraindividual comparison of MRI versus MDCT for endoleak detection after endovascular repair of abdominal aortic aneurysms. Eur Radiol 2009; 19: 1223-31.

65. Habets J, Zandvoort HJ, Reitsma JB, et al. Magnetic resonance imaging is more sensitive than computed tomography angiography for the detection of endoleaks after endovascular abdominal aortic aneurysm repair: a systematic review. Eur J Vasc Endovasc Surg 2013; 45: 340-50.

66. Serafin Z, Strześniewski P, Lasek W, et al. Time-resolved imaging of contrast kinetics does not improve performance of follow-up MRA of embolized intracranial aneurysms. Med Sci Monit 2012; 18: MT60-5.

67. Hallett RL, Ullery BW, Fleischmann D. Abdominal aortic aneurysms: pre- and post-procedural imaging. Abdom Radiol 2018; 43: 1044-66.
68. van der Laan MJ, Bakker CJ, Blankensteijn JD, et al. Dynamic CE-MRA for endoleak classification after endovascular aneurysm repair. Eur J Vasc Endovasc Surg 2006; 31: 130-5.

69. Lookstein RA, Goldman J, Pukin L, et al. Time-resolved magnetic resonance angiography as a noninvasive method to characterize endoleaks: initial results compared with conventional angiography. J Vasc Surg 2004; 39: 27-33.

70. Wieners G, Meyer F, Halloul Z, et al. Detection of type II endoleak after endovascular aortic repair: comparison between magnetic resonance angiography and blood-pool contrast agent and dual-phase computed tomography angiography. Cardiovasc Intervent Radiol 2010; 33: 1135-42.

71. Hope TA, Zarins CK, Herfkens RJ. Initial experience characterizing a type I endoleak from velocity profiles using time-resolved three-dimensional phase-contrast MRI. J Vasc Surg 2009; 49: 1580-4.

72. Van Bogerijen GHW, van Herwaarden JA, Conti M, et al. Importance of dynamic aortic evaluation in planning TEVAR. Ann Cardiothor Surg 2014; 3: 300-6.

73. Sakata M, Takehara Y, Katahashi K, et al. Hemodynamic analysis of endoleaks after endovascular abdominal aortic aneurysm repair by using 4-dimensional flow-sensitive magnetic resonance imaging. Circ J 2016; 80: 1715-25.

74. van Bogerijen GHW, van Herwaarden JA, Conti M, et al. Importance of dynamic aortic evaluation in planning TEVAR. Ann Cardiothorac Surg 2014; 3: 300-6.

75. Wachowski M, Polguj M, Ścibór J, et al. Ischaemia of the medullary cone after stent-graft implantation in a patient with abdominal aortic aneurysm - a case study. Videosurgery Miniinv 2018; 13: 116-21.

76. Bangalore S, Bhatt DL. Femoral arterial access and closure. Circulation 2011; 124: e147-56.

77. Armerding MD, Rubin GD, Beaulieu CF, et al. Aortic aneurysmal disease: assessment of stent-graft treatment-CT versus conventional angiography. Radiology 2000; 215: 138-46.

78. Leszczyński J, Macioch W, Chudziński W, et al. Late type III endoleak after thoracic endovascular aneurysm repair and previous infrarenal stent graft implantation - a case report and review of the literature. Videosurgery Miniinv 2017; 12: 320-4.

79. Garg T, Baker LC, Mell MW. Postoperative surveillance and longterm outcomes after endovascular aneurysm repair among medicare beneficiaries. JAMA Surg 2015; 150: 957-63.

80. Dias NV, Riva L, Ivancev K, et al. Is there a benefit of frequent CT follow-up after EVAR? Eur I Vasc Endovasc Surg 2009; 37: 425-30.

81. Nordon IM, Karthikesalingam A, Hinchliffe RJ, et al. Secondary interventions following endovascular aneurysm repair (EVAR) and the enduring value of graft surveillance. Eur J Vasc Endovasc Surg 2010; 39: 547-54.

Received: 6.07.2018, accepted: 21.08.2018. 